\title{
Increased glucose variability is associated with major adverse events in patients with infective endocarditis undergo surgical treatment
}

\author{
Mengya Liang $^{1 \# \wedge}$, Mai Xiong" ${ }^{1 \#}$, Yi Zhang ${ }^{1}$, Jiantao Chen $^{1}$, Kangni Feng ${ }^{1}$, Suiqing Huang ${ }^{2}$, Zhongkai Wu $^{1}$ \\ ${ }^{1}$ Department of Cardiac Surgery, the First Affiliated Hospital, Sun Yat-Sen University, Guangzhou, China; ${ }^{2}$ Assisted Circulatory Laboratory of \\ Health Ministry, Sun Yat-sen University, Guangzhou, China \\ Contributions: (I) Conception and design: M Liang, Z Wu; (II) Administrative support: M Xiong; (III) Provision of study materials or patients: Y \\ Zhang, S Huang; (IV) Collection and assembly of data: J Chen, K Feng; (V) Data analysis and interpretation: M Liang, Y Zhang; (VI) Manuscript \\ writing: All authors; (VII) Final approval of manuscript: All authors. \\ \#These authors contributed equally to this work. \\ Correspondence to: Zhongkai Wu. The Department of Cardiac Surgery, The First Affiliated Hospital, Sun Yat-sen University, \#58, Zhongshan 2nd \\ Road, Guangzhou 510080, China. Email: wuzhk@mail.sysu.edu.cn.
}

\begin{abstract}
Background: This study aimed to investigate the prognostic value of glucose variability $(\mathrm{GV})$ in predicting postoperative major adverse events (MAEs) in patients with infective endocarditis (IE) who underwent surgical treatment.

Methods: This retrospective observational study included a total of 381 consecutive patients who underwent surgical treatment in our institution from October 2007 to August 2019. The MAEs included allcause death, stroke, myocardial infarction, acute heart failure, IE recurrence, acute renal failure and sepsis. Postoperative GV in the first 24 hours was measured by the mean 24-hour glucose, standard deviation, coefficient of variation (CV) and mean amplitude of glycemic excursions. Univariate and multivariate logistic regression analyses were performed to identify the independent association of GV with MAEs.

Results: Of the 381 patients, 79 (20.7\%) developed MAEs. The 30-day mortality of the overall study cohort was $5.23 \%$. The multivariate logistic regression analysis indicated that 24-hour GV, measured as the $\mathrm{CV}$ [odds ratio $(\mathrm{OR})=1.49,95 \% \mathrm{CI}, 1.23-3.57, \mathrm{P}=0.012$ ], was significantly associated with MAEs in IE patients. For every $10 \%$ increase in 24 -hour CV, there was a $49 \%$ increase in the risk of MAEs. Furthermore, compared to patients in the low tertile of GV, patients in the top tertile of 24-hour GV had a higher 30-day mortality and an increased incidence of heart failure and hemodialysis as well as longer ventilation support.
\end{abstract}

Conclusions: The results of this retrospective investigation demonstrated that increased GV measured by $\mathrm{CV}$ is an independent predictor of postoperative MAEs in patients undergoing surgical treatment for IE.

Keywords: Infective endocarditis (IE); major adverse events (MAEs); glucose variability (GV)

Submitted Aug 19, 2020. Accepted for publication Nov 20, 2020.

doi: $10.21037 /$ jtd-20-2692

View this article at: http://dx.doi.org/10.21037/jtd-20-2692

\footnotetext{
^ ORCID: 0000-0003-1879-8543.
} 


\section{Introduction}

The treatment of infective endocarditis (IE) remains challenging for cardiologists and cardiac surgeons in the new century despite recent advances in medicine over the past decades. According to recent reports, complications and mortality rates remain high in IE patients, even in the developed world (1). The major adverse events (MAEs) that occur in postoperative patients include all-cause death, stroke, myocardial infarction, acute heart failure, IE recurrence, acute renal failure and sepsis. These MAEs are closely associated with patient prognosis and mortality. Thus, the identification of a biomarker at the initial evaluation that could predict MAEs in patients after surgical intervention would be very valuable and helpful for clinical care.

The disease process of IE is usually accompanied by a systemic inflammatory response, which consequently causes aberrant fluctuations in serum glucose levels. Disturbances of glucose metabolism are frequently seen in critically ill patients with IE. Sustained hyperglycemia is an established risk factor for adverse ICU outcomes following cardiovascular surgery (2). Glucose variability (GV) is a relatively novel indicator which refers to swings in blood glucose levels (3), it has a broader meaning than glucose numerical value itself because it alludes to blood glucose oscillations that occur throughout the day, not only including hyperglycemia but also hypoglycemic periods and postprandial increases, as well as blood glucose fluctuations that occur at the same time period. It has been demonstrated that elevated GV is associated with MAEs after coronary artery bypass grafting (CABG) (4). Whether postoperative $\mathrm{GV}$ has important value in predicting the prognosis of IE patients remains unknown and needs further investigation. The aim of the present study was to retrospectively investigate the prognostic value of $\mathrm{GV}$ in predicting postoperative MAEs in IE patients. We present the following article in accordance with the STROBE reporting checklist (available at http://dx.doi.org/10.21037/ jtd-20-2692).

\section{Methods}

\section{Patient cohort}

The study was conducted in accordance with the Declaration of Helsinki (as revised in 2013). This retrospective observational study was approved by the institutional review board of the First Affiliated Hospital of Sun Yat-sen University (IRB-FAH-SYSU). The need for informed consent was waived due to the retrospective nature of the study and the method of data extraction of medical records. The data for this retrospective study were extracted from electronic patient medical records using the hospital information system of the First Affiliated Hospital of Sun Yat-sen University (HIS/FAHSYSU) and the intensive care medical record system of the First Affiliated Hospital of Sun Yat-sen University (ICMRS/FAHSYSU).

Between October 2007 and August 2019, a total of 583 consecutive patients who received a diagnosis of definite IE in the First Affiliated Hospital of Sun Yat-sen University were screened for eligibility. Patients were excluded if they did not undergo surgical treatment, if they had incomplete postoperative glycemic records, or if they developed severe hypoglycemic events preoperatively. In total, 381 patients with IE who underwent surgical treatment at our institution were enrolled in the present study (Figure 1). IE was diagnosed according to the modified Duke criteria (5), and vegetation was classified by transthoracic or transesophageal echocardiography. Blood culture results were obtained from bacteriology laboratories, and antibiotics were administered according to results of antibiotic susceptibility testing.

\section{Surgical techniques}

Candidates for surgical treatment were determined in accordance with the ESC Guidelines for the management of IE (6). All surgeries were performed through median sternotomy, with cardiopulmonary bypass $(\mathrm{CPB})$ in mild systemic hypothermia $\left(32-34{ }^{\circ} \mathrm{C}\right)$ and antegrade crystalloid cardioplegia. The surgical procedure was performed according to the predisposing heart disease (Table 1). Either valve replacement or valve repair procedures were performed if the native valves were affected (Table 2).

\section{Protocol for postoperative glucose monitoring and control}

After admission to the cardiothoracic intensive care unit of the First Affiliated Hospital of Sun Yat-sen University, patients received frequent glucose monitoring in accordance with the First Affiliated Hospital of Sun Yat-sen University Cardiovascular Surgical Intensive Care Unit Blood Glucose Monitoring and Control Protocol (https://cdn.amegroups. cn/static/public/JTD-20-2692-1.pdf). Continuous intravenous insulin infusion was administered if required, which aims for a target glucose range from $4-10 \mathrm{mmol} / \mathrm{L}$ $(72-180 \mathrm{mg} / \mathrm{dL})$. Of 381 patients, $199(52.2 \%)$ required postoperative insulin infusion, and 320 patients $(84.1 \%)$ 


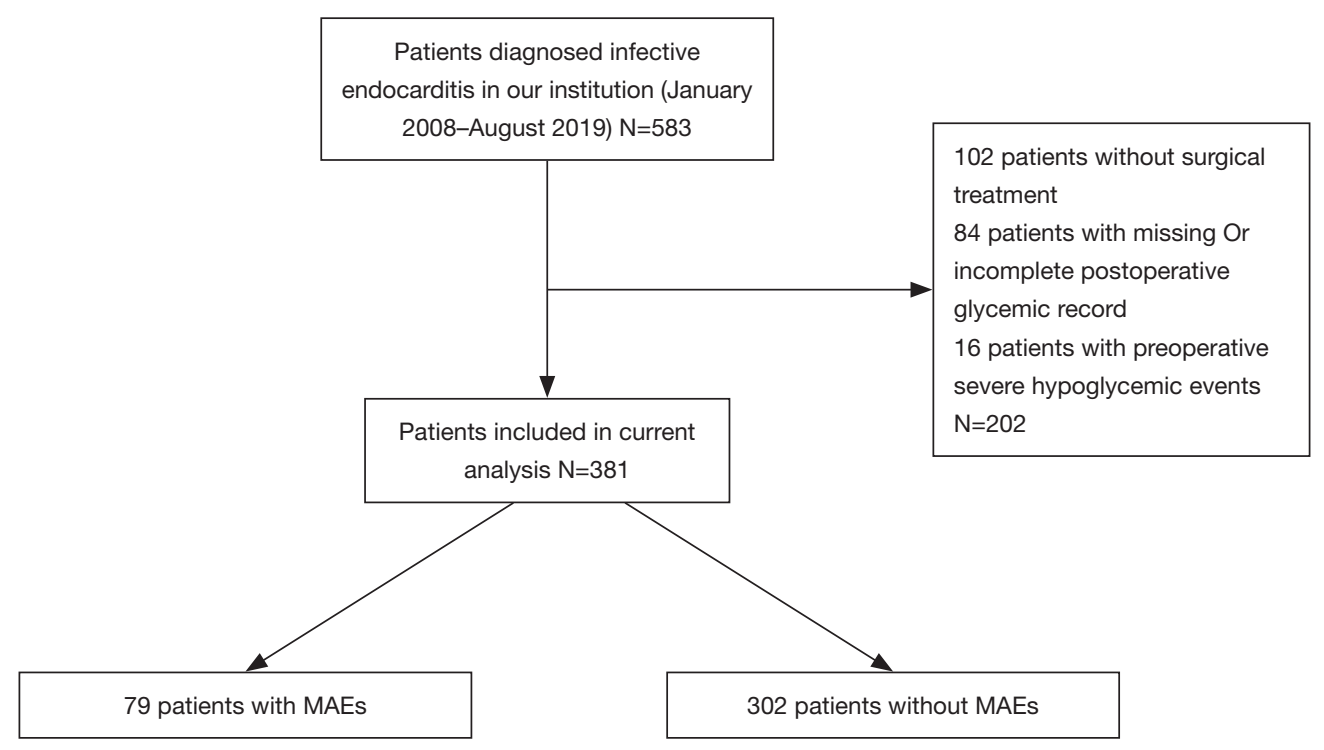

Figure 1 Flow diagram of the patient cohort. MAEs, major adverse events.

achieved the target postoperative glucose levels of $4-10 \mathrm{mmol} / \mathrm{L}$. The glucose levels within the first 24 hours postoperatively were collected for further glycemic variability calculations. Episodes of hypoglycemic events (defined as a glucose level $<70 \mathrm{mg} / \mathrm{dL})$, severe hypoglycemic events $(<50 \mathrm{mg} / \mathrm{dL})$ and hyperglycemic events $(>180 \mathrm{mg} / \mathrm{dL})$ were recorded.

\section{Glycemic variability parameters}

Glycemic variability within the first 24 hours in the ICU was evaluated using the following parameters: (I) mean 24-hour glucose, defined as the mean of the glucose levels recorded within the first 24 hours. (II) standard deviation (SD), defined as the SD of all blood glucose readings in a patient within the first 24 hours postoperatively. (III) the coefficient of variability (CV), defined as the SD of blood glucose divided by the mean and expressed as a percentage. Although the SD can reflect the variation of glucose level swinging around the mean glucose, it is highly influenced by the mean glucose, i.e., patients with a higher mean glucose will have a higher SD. By dividing SD by mean glucose, CV is designed to adjust and normalize the variability among patients with different mean glucose levels and provide a more accurate and comparable metric of GV (3). (IV) the mean amplitude of glycemic excursions (MAGE) captures meal-time glucose fluctuations and is defined as the mean of blood glucose fluctuations larger than one SD of the glucose measurements (3).

\section{Outcome assessment}

The primary endpoint was the incidence of postprocedural MAEs occurring between the first day and the 30th day after open-heart surgery. This composite endpoint included 30-day death from all causes, postoperative neurological events, acute heart failure, IE recurrence, acute renal failure requiring dialysis, sepsis and prolonged ventilation.

\section{Statistical analysis}

All statistical analyses were performed using SPSS version 22.0 for Windows (SPSS Inc., Chicago, IL, USA). Continuous variables were tested for normal distribution by the Kolmogorov-Smirnov test. Data are presented as the mean \pm SD or median (25 th percentile- 75 th percentile). Student's $t$ test was used to compare normally distributed data between the groups. The Mann-Whitney U test was used to compare the nonnormally distributed data. Categorical variables were compared with Fisher's exact test.

For the multivariable logistic regression, a previously reported purposeful selection model-building strategy was used to create a parsimonious model that included preoperative and operative factors (7). Preoperative and operative candidate covariates with a $\mathrm{P}$ value less than 0.10 in the univariate analysis or covariates reported in previous literature to be associated with adverse events in IE patients 
Table 1 Preoperative characteristics of patients with and without postoperative major adverse events

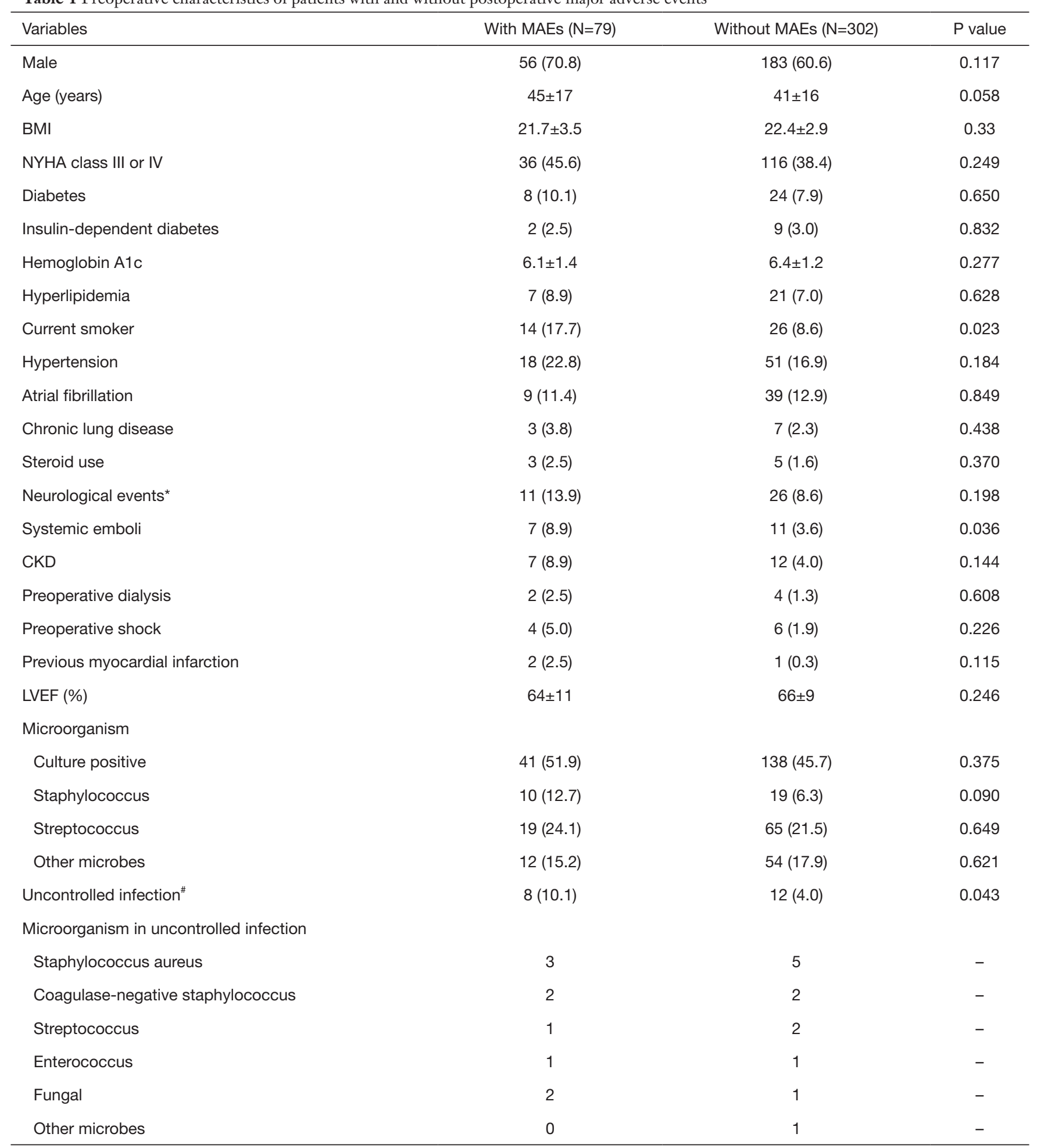

Table 1 (continued) 
Table 1 (continued)

\begin{tabular}{|c|c|c|c|}
\hline Variables & With MAEs (N=79) & Without MAEs (N=302) & $P$ value \\
\hline Rheumatic heart disease & $7(8.9)$ & $18(6.0)$ & 0.442 \\
\hline Degenerative & $37(46.8)$ & $155(51.3)$ & 0.528 \\
\hline Congenital heart disease & $8(10.1)$ & $25(8.3)$ & 0.653 \\
\hline Lead involvement & $0(0)$ & $2(0.6)$ & 1.000 \\
\hline Other diseases & $25(31.6)$ & $99(32.7)$ & 0.893 \\
\hline
\end{tabular}

Data are expressed as mean \pm standard deviation and $n(\%)$. *Postoperative neurological events include the following events: stroke, embolic infarction, abscess and subarachnoid bleeding. "Uncontrolled infection: endocarditis refractory to sufficient antibiotics therapy for 2 weeks. MAEs, major adverse events; CKD, chronic kidney disease; NYHA, New York Heart Association; LVEF, left ventricular ejection fraction.

were included in the multivariable logistic regression. Variables not contributing to the model $(\mathrm{P}>0.05)$ were individually removed, and the model was refitted at each step to create the most parsimonious model. Model fit was assessed and determined by the Hosmer-Lemeshow test. A series of logistic regression models was created with nine preoperative and operative variables and 24-hour GV to determine the correlation between 24-hour GV and MAEs in the IE patient population. For all tests, $\mathrm{P}<0.05$ was considered to indicate statistical significance.

\section{Results}

\section{Baseline characteristics and intraoperative data}

The preoperative characteristics and intraoperative parameters of the patients with and without MAEs are listed in Tables 1 and 2. Most preoperative parameters were identical between patients with or without MAEs, except for current smoking and the incidence of systemic emboli ( $\mathrm{P}=0.023$ and $\mathrm{P}=0.036$, respectively). It is noteworthy that the incidence of diabetes, insulin-dependent diabetes and hemoglobin A1c were not significantly different between these two groups. The uncontrolled infection rate was significantly higher in patients with MAEs than in those without MAEs $(\mathrm{P}=0.043)$.

\section{Episodes of MAEs in the study cohort}

The total number of episodes of MAEs was 117 (Table 2). Of the entire study cohort, 20 patients died within 30 days postoperatively. The 30-day mortality of the entire study cohort was $5.23 \%$. Additionally, 31 patients developed heart failure in the postoperative phase, 21 patients needed hemodialysis in the ICU, 24 patients required prolonged ventilator support, 11 patients experienced neurological events, and sepsis and IE recurrence were found in 7 and 3 patients, respectively. Furthermore, the study population was divided into two groups of high and low postoperative glycemic fluctuation according to GV levels (Table 3). High $\mathrm{GV}$ is defined as the top tertile of 24-hour postoperative $\mathrm{GV}$ measured by $\mathrm{CV}$, and low $\mathrm{GV}$ is the bottom tertile of 24-hour postoperative GV measured by CV. As demonstrated in Table 3, compared with patients with low GV, patients with high 24-hour GV had a higher 30-day mortality $(\mathrm{P}=0.019)$. Moreover, patients in the top tertile of 24-hour GV exhibited a higher incidence of heart failure, hemodialysis and prolonged ventilation support than patients in the low tertile of 24-hour GV ( $\mathrm{P}=0.021,0.006$ and 0.003 , respectively). The overall episodes of MAEs and the number of cases with MAEs were found to be significantly higher in patients with high 24-hour GV than in those with low 24-hour GV.

\section{Glycemic variability}

Measures of postoperative glycemic variability are listed in Table 4. Interestingly, there were no significant differences in the mean 24-hour glucose levels between the groups with and without MAEs $(\mathrm{P}=0.625)$. However, patients with MAEs had significantly higher SD, CV and MAGE glucose levels than patients without MAEs $(\mathrm{P}<0.001, \mathrm{P}<0.001$ and $\mathrm{P}=0.001$, respectively), suggesting that the measures of glycemic fluctuation are more sensitive than the mean 
Table 2 Intraoperative and postoperative parameters of patients with and without postoperative major adverse events

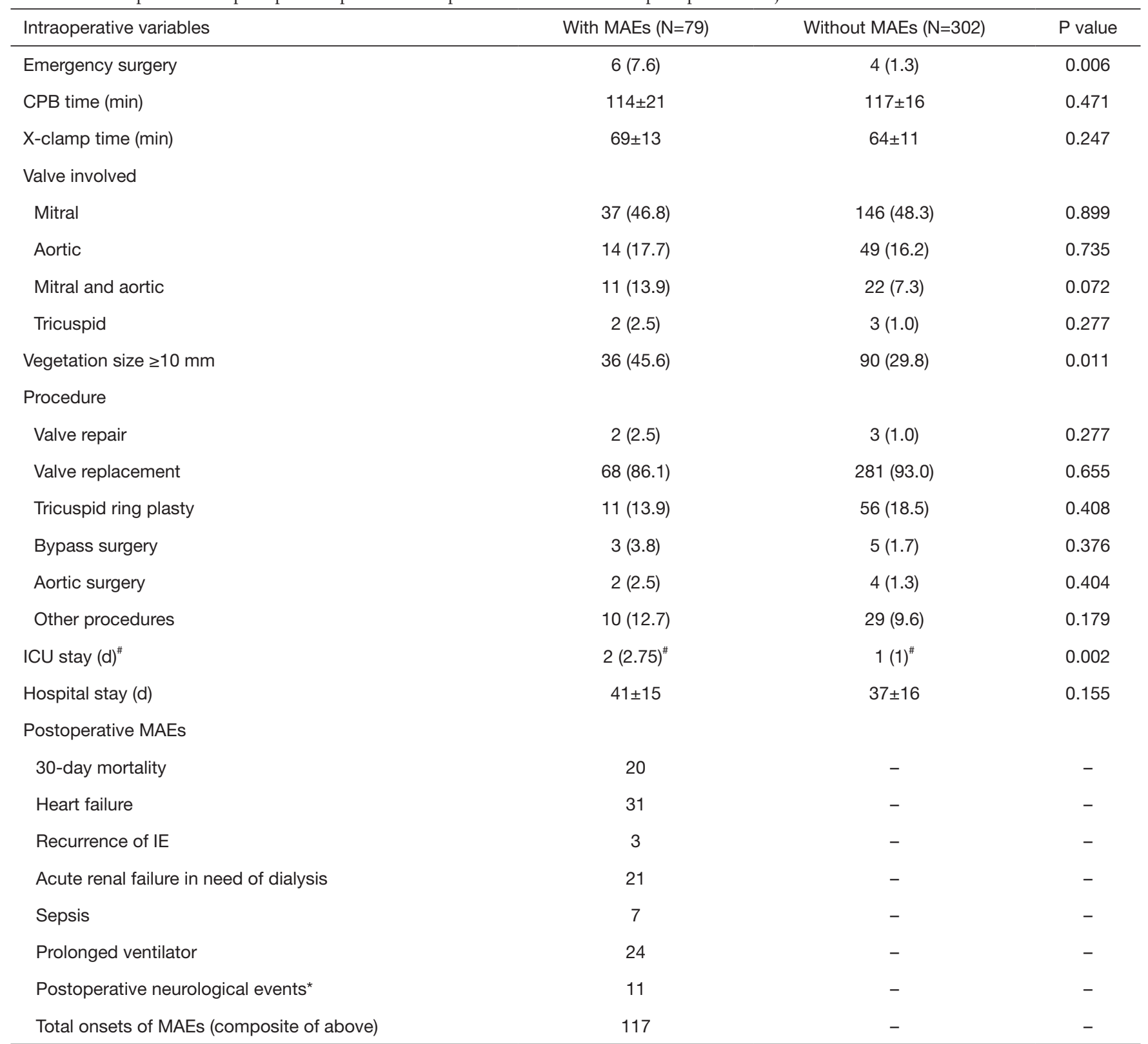

Data are expressed as mean \pm standard deviation and $\mathrm{n}(\%)$. ${ }^{\text {HData }}$ are expressed as median $\left(25^{\text {th }}-75^{\text {th }}\right.$ percentile); ${ }^{*}$ Postoperative neurological events include the following events: stroke, embolic infarction, abscess and subarachnoid bleeding. MAEs, major adverse events; CPB, cardiopulmonary bypass; ICU, intensive care unit; X-clamp time, cross-clamp time.

glucose level. The number of patients who required postoperative insulin infusion were not different between the two groups $(\mathrm{P}=0.0579)$. Moreover, compared with those without MAEs, patients with MAEs had more recorded episodes of hypoglycemic events, severe hypoglycemic events and hyperglycemic events $(\mathrm{P}<0.001, \mathrm{P}=0.009$ and $\mathrm{P}<0.001$, respectively).

\section{Multivariable logistic regression to predict postoperative MAEs}

Nine preoperative and intraoperative candidate covariates (Table 5) were included in the parsimonious model for multivariable logistic regression. After progressively adjusting for these covariates, 24-hour GV measured as CV (odds ratio 1.49, 95\% confidence interval, 1.23-3.57, 
Table 3 Postoperative major adverse events (MAEs) in patients with high and low postoperative glycemic fluctuation

\begin{tabular}{|c|c|c|c|c|}
\hline Variables & High GV (N=127) & Intermediate GV (N=127) & Low GV (N=127) & $P$ value \\
\hline Heart failure & $16(12.6)$ & $10(7.9)$ & $5(3.9)$ & 0.021 \\
\hline Recurrence of IE & $0(0)$ & $2(1.5)$ & $1(0.8)$ & 0.992 \\
\hline Acute renal failure in need of dialysis & $15(11.8)$ & $3(2.3)$ & $3(2.3)$ & 0.006 \\
\hline Prolonged ventilator & $14(11.0)$ & $6(4.7)$ & $2(1.6)$ & 0.003 \\
\hline Postoperative neurological events* & $6(4.7)$ & $3(2.3)$ & $2(1.6)$ & 0.172 \\
\hline Total onset of MAEs (composite of above) & $64(50.4)$ & $36(28.3)$ & $18(14.2)$ & $<0.001$ \\
\hline Cases with MAEs & $51(40.2)$ & $22(17.3)$ & $13(10.2)$ & $<0.001$ \\
\hline
\end{tabular}

High glucose variability is the top tertile of 24-hour postoperative glucose variability measured by coefficient of variation. Intermediate GV is the middle tertile of 24-hour postoperative glucose variability measured by coefficient of variation. Low glucose variability is the bottom tertile of 24-hour postoperative glucose variability measured by coefficient of variation. Data are expressed as mean \pm standard deviation and $\mathrm{n}(\%)$. *Postoperative neurological events included the following events occurred in central nervous system: stroke, embolic infarction, abscess and subarachnoid bleeding. GV, glucose variability; MAEs, major adverse events.

Table 4 Measures of postoperative glycemic fluctuation between patients with and without postoperative major adverse events

\begin{tabular}{|c|c|c|c|}
\hline Variables & With MAEs (N=79) & Without MAEs (N=302) & $P$ value \\
\hline Standard deviation of 24-h glucose & $2.74(2.29)$ & $1.92(1.26)$ & $<0.001$ \\
\hline Coefficient of variation of $24-\mathrm{h}$ glucose & $28(16.0)$ & $19(10.0)$ & $<0.001$ \\
\hline Mean amplitude of glycemic excursions (MAGE) of 24-h glucose & $4.2(4.11)$ & $3.08(2.15)$ & 0.001 \\
\hline Cases with hypoglycemic events ( $<70 \mathrm{mg} / \mathrm{dL})$ & $11(13.92)$ & $1(0.3)$ & $<0.001$ \\
\hline Cases with severe hypoglycemic events ( $<50 \mathrm{mg} / \mathrm{dL})$ & $3(3.80)$ & $0(0)$ & 0.009 \\
\hline Cases with hyperglycemic events (>180 mg/dL) & $72(91.14)$ & $300(99.34)$ & $<0.001$ \\
\hline
\end{tabular}

Variables of postoperative glycemic fluctuation are presented as medians (interquartile range), the enumeration data are presented as $\mathrm{n}$ (\%). Mean 24-h glucose, Standard deviation of 24-h glucose, coefficient of variation of 24-h glucose and mean amplitude of glycemic excursions of 24-h glucose are presented as mmol/dL. MAEs, major adverse events.

$\mathrm{P}=0.012$ ) was significantly associated with postoperative MAEs in IE patients. In the current study, the OR for 24-hour GV reflects a $10 \%$ change in GV measured as $\mathrm{CV}$; thus, the current result exhibited in our patient cohort shows that, for every $10 \%$ increase in 24 -hour $\mathrm{CV}$, there was a $49 \%$ increase in the incidence of MAEs in IE patients.

\section{Discussion}

In the past decade, there have been quite a few advancements in diagnostic and therapeutic approaches for
IE; however, IE remains one of the most life-threatening cardiothoracic diseases and is associated with high mortality and morbidity (8-10). A better understanding of the association between clinical markers and the outcome is crucial to treatment decisions and prognostic evaluations. Although a small number of clinical markers were reported to not be responsible for the mortality and occurrence of MAEs in this population $(5,11,12)$, the role of glucose levels and variability has not yet been investigated. Glucose levels are frequently monitored in the ICU, which makes $\mathrm{GV}$ a simple and cost-effective indicator for the prognostic 
Table 5 Multivariable logistic regression of postoperative 24-h glucose variability and postoperative major adverse events

\begin{tabular}{llll}
\hline Variables & OR & $95 \% \mathrm{Cl}$ & $\mathrm{P}$ value \\
\hline 24-h glucose variability and MAEs & & & $0.98-1.21$ \\
Age & 1.08 & $0.84-1.02$ & 0.345 \\
Hemoglobin A1c & 0.92 & $0.82-5.42$ & 0.114 \\
Systemic emboli & 2.63 & $0.93-2.85$ & 0.193 \\
Staphylococcus infection & 1.18 & $0.63-6.94$ & 0.455 \\
Uncontrolled infection & 2.51 & $0.77-22.10$ & 0.677 \\
Prosthetic valve endocarditis & 5.81 & $1.285-12.51$ & 0.025 \\
Emergency surgery & 5.27 & $1.12-3.68$ & 0.043 \\
Vegetation size $\geq 10$ mm & 1.51 & $0.88-5.25$ & 0.237 \\
Mitral and aortic involvement & 1.78 & $1.23-3.57$ & 0.012 \\
24-h glucose variability & 1.49 & & 0.010 \\
Constant & 0.02 & & \\
\hline
\end{tabular}

MAEs, major adverse events.

evaluation of IE patients. A meta-analysis of twelve retrospective studies reported that GV had a statistically significant association with high mortality in patients who underwent general surgery in the ICU (13). However, to date, no study has reported the efficacy of glycemic variability for predicting outcomes of patients with IE. The main finding of our study is that increased GV measured by $\mathrm{CV}$ is associated with MAEs and is also an independent predictor of postoperative MAEs, and for every $10 \%$ increase in 24 -hour $\mathrm{CV}$, there is a $49 \%$ increase in the incidence of MAEs.

We retrospectively reviewed a study cohort of $381 \mathrm{IE}$ patients who underwent surgical treatment in our institution in the past decade. Of these 381 patients, 79 patients (20.7\%) developed MAEs postoperatively. The univariate analysis indicated that postoperative MAEs were associated with age, systemic emboli, staphylococcus infection, uncontrolled infection, prosthetic valve endocarditis, emergency surgery, vegetation size $\geq 10 \mathrm{~mm}$ and both mitral and aortic involvement. In the previous studies, these risk factors were reported to be associated in varying degrees with the outcomes of IE patients $(12,14,15)$. On the other hand, although in the current study hemoglobin A1c did not show any significant difference between patients with and without MAEs, the study by Subramaniam et al. demonstrated that preoperative hemoglobin A1c identifies the risk for postoperative glycemic variability and had somewhat of an effect on the incidence of MAEs following bypass surgery (16); thus, we also included hemoglobin A1c in our model to minimize its influence on postoperative glycemic variability. With these risk factors entered into the aforementioned parsimonious model and after adjusting for nine covariances in the multivariable logistic regression, the 24-hour glycemic variability measured as CV was identified as an independent predictor for postoperative MAEs. We also found that, compared to patients with low GV, patients with high 24-hour GV had a higher 30-day mortality and increased incidence of heart failure and hemodialysis as well as longer ventilation support.

The findings of the present study are clinically relevant because the study cohort was under tight glucose control with an insulin infusion following the aforementioned protocol in the ICU, which indicated that a more stable and lower GV would be measured. Nonetheless, the current results revealed that increased GV rather than hyperglycemia was more detrimental to IE patients. Several previous studies may provide some evidence to explain why $\mathrm{GV}$ is important in predicting clinical outcomes. Liu and colleagues demonstrated that oscillating high glucose could be more detrimental and toxic to human coronary artery endothelial cells than persistent high glucose, probably due to the enhancement of oxidative stress and cellular apoptosis induced by frequent glucose swings (17). Another study by Ying et al. reported that blood GV can worsen cardiac 
Table 6 Measures of glycemic variability between patients underwent intensive glucose control ${ }^{\#}$ and conventional glucose control*

\begin{tabular}{|c|c|c|c|}
\hline Variables & With intensive glucose control $(\mathrm{N}=32)$ & With conventional glucose control $(\mathrm{N}=349)$ & $P$ value \\
\hline Standard deviation of 24-h glucose & $3.19(2.7)$ & $2.36(1.22)$ & 0.33 \\
\hline Coefficient of variation of $24-\mathrm{h}$ glucose & $26(19.0)$ & $24(14.0)$ & 0.61 \\
\hline $\begin{array}{l}\text { Mean amplitude of glycemic excursions } \\
\text { (MAGE) of 24-h glucose }\end{array}$ & $3.67(3.1)$ & $3.33(2.55)$ & 0.47 \\
\hline
\end{tabular}

Variables of postoperative glycemic fluctuation are presented as medians (interquartile range), the enumeration data are presented as $\mathrm{n}$ (\%). Mean 24-h glucose, Standard deviation of 24-h glucose, Coefficient of variation of 24-h glucose and Mean amplitude of glycemic excursions of 24-h glucose are presented as mmol/dL. "Intensive glucose control: glucose levels are monitored and insulin infusion is used to achieve a rigorous glucose target (72 to $144 \mathrm{mg} / \mathrm{dL}$ ); ${ }^{*}$ Conventional glucose control: glucose levels are monitored and insulin infusion is used to achieve a broad glucose target (72 to $180 \mathrm{mg} / \mathrm{dL})$.

fibrosis, possibly through mechanisms involving oxidative stress, by inhibiting the AKT signaling pathway (18). Furthermore, in diabetic patients, compared to an aberrant mean glucose level, oscillating glucose levels were more deleterious to endothelial function and produced more oxidative stress (19). The above studies suggest that glycemic fluctuation results in endothelial impairment and metabolic disorders and causes further multiple organ dysfunction.

In 2009, Finfer et al. published a landmark glucose control study and demonstrated that intensive glucose control increased mortality among adults in the ICU comparing to conventional control (20). Afterwards the majority of guidelines concerning ICU glycose management recommended that insulin infusion should be used to achieve a broader glucose target (less than $180 \mathrm{mg} / \mathrm{dL}$ ) other than previous rigorous target (less than $144 \mathrm{mg} / \mathrm{dL}$ ). Therefore, the protocol of glucose management in our cardiovascular ICU was updated in August 2010 (https://cdn.amegroups.cn/static/public/JTD20-2692-1.pdf). Apparently, the patients in the present study cohort who were hospitalized between 2007 to 2010 with initial glucose level between 144 to $180 \mathrm{mg} / \mathrm{dL}$ received more intensive glucose checks and earlier intervention of insulin than those who were admitted afterwards and managed following the updated protocol. To identify whether the changes in protocol of glucose management have influence on the findings of current study, we performed a statistical comparison between the patients who were admitted before update of protocol and afterwards. As showed in Table 6, Only a small proportion of patients (32 cases, $8.3 \%$ ) were admitted to our institution before protocol update whereas others (349 cases) were admitted afterwards. The metrics of GV did not show any statistically difference between patients treated with different strategies, which possibly was due to the small case number treated with intensive glucose control. Therefore, we believed the findings from current study is still clinically significant and applicable even though the criteria of glucose target and insulin infusion was changed during the study period.

There are several limitations in this study. First, in clinical practice, glucose monitoring in the ICU was not performed at the same interval, and critically ill patients and patients in need of intravenous insulin infusion were more likely to receive more frequent glucose measurements than other patients, which probably resulted in bias in the GV calculation. Because in accordance with the protocol of glucose management in current study, the patients whose blood glucose $>144 \mathrm{mg} / \mathrm{dL}$ or in need of insulin infusion are able to receive a higher frequency of glucose measurements, patients with high frequency of measurements in the first 24 hours are likely to have a more stable and accurate standard deviation than those with less measurements. Given standard deviation as an important variable in calculating $\mathrm{CV}$ and MAGE, it's likely that there is a smaller bias of CV and MAGE calculation in patients with more measurements than those with less measurements. Second, the study population comprised patients with different underlying heart diseases who underwent different types of surgical procedures, e.g., valve surgeries and congenital heart surgeries. It is likely that the underlying disease and type of surgical treatment would have different influences on the outcome of the patients. Further investigation 
focused on the response of individual populations to specific surgical treatments is warranted. Third, a small number of patients without complete perioperative glycemic records were excluded from this study, especially because hemoglobin A1c is not a mandatory preoperative test in our institution; however, we still believe that the evidence and data from the present cases are sufficient and convincing to support the current conclusion. Fourth, the available metrics of $\mathrm{GV}$ in present study, i.e., $\mathrm{SD}, \mathrm{CV}$ and MAGE, cannot provide information that accounts for the time-duration of particular glucose value. We have noted continuous glucose monitoring (CGM), a novel continuous glucose monitoring technique, can offer better information of time-duration of glucose values and enable rapid and sensitive modification of insulin infusion. However, due to the consideration of cost performance and medical insurance policy, the CGM device is not yet in clinical use in our institution. Considering the findings of current study suggested the potential effectiveness of GV metrics in predicting MAEs in IE population, we believe introducing CGM has good prospects in this population and is deserving further investigation in the future.

\section{Conclusions}

In summary, the results of this retrospective investigation demonstrated that increased $\mathrm{GV}$ measured by $\mathrm{CV}$ is not only associated with MAEs but also an independent predictor of postoperative MAEs in patients undergoing surgical treatment for IE, and for every $10 \%$ increase in $24-$ hour $\mathrm{CV}$, there is a $49 \%$ increase in the risk of MAEs.

\section{Acknowledgments}

The authors are indebted to Dr. Chen-yang Feng, School of Public Health, Sun Yat-sen University, Guangzhou, for excellent technical assistance for statistical work. The grammars of manuscript were checked by a medical writing service provider American Journal Expert. Inc.

Funding: This work was supported by the National Natural Science Funds of China (Grant Number 81400307); and the Natural Science Funds of Guangdong Province (Grant Number 2019A1515010218).

\section{Footnote}

Reporting Checklist: The authors have completed the STROBE reporting checklist. Available at http://dx.doi. org/10.21037/jtd-20-2692

Data Sharing Statement: Available at http://dx.doi. org/10.21037/jtd-20-2692

Conflicts of Interest: All authors have completed the ICMJE uniform disclosure form (available at http://dx.doi. org/10.21037/jtd-20-2692). The authors have no conflicts of interest to declare.

Ethical Statement: The authors are accountable for all aspects of this retrospective observational study in ensuring that questions related to the accuracy or integrity of any part of the work are appropriately investigated and resolved. The study was conducted in accordance with the Declaration of Helsinki (as revised in 2013). This retrospective observational study was approved by the institutional review board of the First Affiliated Hospital of Sun Yatsen University (IRB-FAH-SYSU). The need for informed consent was waived due to the retrospective nature of the study and the method of data extraction of medical records.

Open Access Statement: This is an Open Access article distributed in accordance with the Creative Commons Attribution-NonCommercial-NoDerivs 4.0 International License (CC BY-NC-ND 4.0), which permits the noncommercial replication and distribution of the article with the strict proviso that no changes or edits are made and the original work is properly cited (including links to both the formal publication through the relevant DOI and the license). See: https://creativecommons.org/licenses/by-nc-nd/4.0/.

\section{References}

1. Cahill TJ, Baddour LM, Habib G, et al. Challenges in Infective Endocarditis. J Am Coll Cardiol 2017;69:325-44.

2. Bardia A, Khabbaz K, Mueller A, et al. The Association Between Preoperative Hemoglobin A1C and Postoperative Glycemic Variability on 30-Day Major Adverse Outcomes Following Isolated Cardiac Valvular Surgery. Anesth Analg 2017;124:16-22.

3. DeVries JH. Glucose variability: where it is important and how to measure it. Diabetes 2013;62:1405-8.

4. Clement KC, Suarez-Pierre A, Sebestyen K, et al. Increased Glucose Variability Is Associated With Major Adverse Events After Coronary Artery Bypass. Ann Thorac Surg 2019;108:1307-13.

5. Li JS, Sexton DJ, Mick N, et al. Proposed modifications 
to the Duke criteria for the diagnosis of infective endocarditis. Clin Infect Dis 2000;30:633-8.

6. Habib G, Lancellotti P, Antunes MJ, et al. 2015 ESC Guidelines for the management of infective endocarditis: The Task Force for the Management of Infective Endocarditis of the European Society of Cardiology (ESC). Eur Heart J 2015;36:3075-128.

7. Zhang Z. Model building strategy for logistic regression: purposeful selection. Ann Transl Med 2016;4:111.

8. Thuny F, Giorgi R, Habachi R, et al. Excess mortality and morbidity in patients surviving infective endocarditis. Am Heart J 2012;164:94-101.

9. Fernández-Hidalgo N, Almirante B, Tornos P, et al. Immediate and long-term outcome of left-sided infective endocarditis. A 12-year prospective study from a contemporary cohort in a referral hospital. Clin Microbiol Infect 2012;18:E522-30.

10. Mori M, Bin Mahmood SU, Schranz AJ, et al. Risk of reoperative valve surgery for endocarditis associated with drug use. J Thorac Cardiovasc Surg 2020;159:1262-8.e2.

11. Turak O, Özcan F, İşleyen A, et al. Usefulness of Neutrophil-to-Lymphocyte Ratio to Predict In-hospital Outcomes in Infective Endocarditis. Can J Cardiol 2013;29:1672-8.

12. Wang CY, Wang YC, Yang YS, et al. Microbiological features, clinical characteristics and outcomes of infective endocarditis in adults with and without hemodialysis: A 10year retrospective study in Northern Taiwan. J Microbiol Immunol Infect 2020;53:336-43.

13. Eslami S, Taherzadeh Z, Schultz MJ, et al. Glucose

Cite this article as: Liang M, Xiong M, Zhang Y, Chen J, Feng $\mathrm{K}$, Huang $\mathrm{S}, \mathrm{Wu} \mathrm{Z}$. Increased glucose variability is associated with major adverse events in patients with infective endocarditis undergo surgical treatment. J Thorac Dis 2021;13(2):653-663. doi: $10.21037 /$ jtd-20-2692 variability measures and their effect on mortality: a systematic review. Intensive Care Med 2011;37:583-93.

14. Fernández Guerrero ML, Gonzalez JJ, Goyenechea A, et al. Endocarditis caused by Staphylococcus aureus: A reappraisal of the epidemiologic, clinical, and pathologic manifestations with analysis of factors determining outcome. Medicine (Baltimore) 2009;88:1-22.

15. Thuny F, Beurtheret S, Mancini J, et al. The timing of surgery influences mortality and morbidity in adults with severe complicated infective endocarditis: a propensity analysis. Eur Heart J 2011;32:2027-33.

16. Subramaniam B, Lerner A, Novack V, et al. Increased glycemic variability in patients with elevated preoperative HbA1C predicts adverse outcomes following coronary artery bypass grafting surgery. Anesth Analg 2014;118:277-87.

17. Liu TS, Pei YH, Peng YP, et al. Oscillating high glucose enhances oxidative stress and apoptosis in human coronary artery endothelial cells. J Endocrinol Invest 2014;37:645-51.

18. Ying C, Liu T, Ling H, et al. Glucose variability aggravates cardiac fibrosis by altering AKT signalling path. Diab Vasc Dis Res 2017;14:327-35.

19. Ceriello A, Esposito K, Piconi L, et al. Oscillating glucose is more deleterious to endothelial function and oxidative stress than mean glucose in normal and type 2 diabetic patients. Diabetes 2008;57:1349-54.

20. Finfer S, Chittock DR, Su SY, et al. Intensive versus conventional glucose control in critically ill patients. $\mathrm{N}$ Engl J Med 2009;360:1283-97. 